
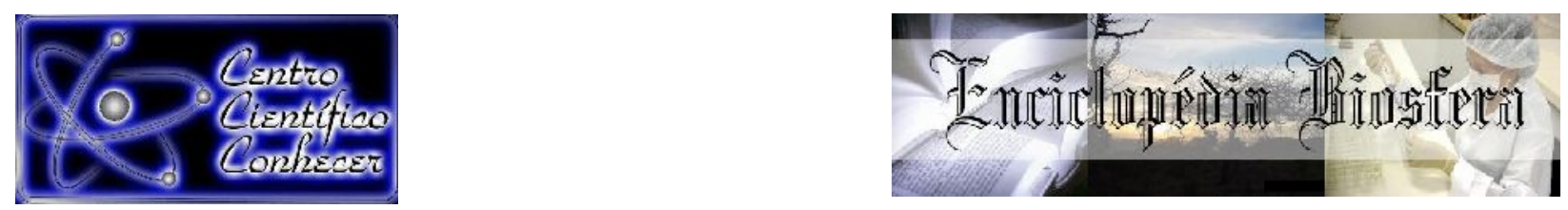

\title{
CONSTRUÇÃO DE UM PROTÓTIPO DE TORRE DE RESFRIAMENTO DE ÁGUA
}

Brunna Coutinho Leal ${ }^{1}$, Vinícius Romano Gisoldi ${ }^{2}$, Marcelo Bacci da Silva ${ }^{3}$

1,2 Graduandos em Engenharia Química da Universidade Federal do Triângulo Mineiro, Uberaba, Brasil.

3 Professor Doutor do Departamento de Engenharia Química da Universidade

Federal do Triângulo Mineiro, Uberaba, Brasil.

(bacciuftm@gmail.com)

\section{Recebido em: 15/11/2021 - Aprovado em: 15/12/2021 - Publicado em: 30/12/2021 \\ DOI: 10.18677/EnciBio_2021D20}

trabalho licenciado sob licença Creative Commons Attribution-NonCommercial-NoDerivatives 4.0 International License

\section{RESUMO}

O tratamento de água em sistemas de resfriamento afeta a eficácia da produção e a vida útil dos equipamentos envolvidos. Em tais processos, para suprir a alta demanda hídrica, a água é reaproveitada. Para projetar equipamentos que envolvem trocas térmicas, equações de balanços de massa e energia são utilizadas e os coeficientes de transferência de calor e de massa devem ser estimados com precisão razoável, permitindo atingir os gradientes de temperatura exigidos para o bom desempenho dos equipamentos. O objetivo do estudo foi construir e analisar o desempenho de uma torre de resfriamento de água para uso nas aulas práticas do curso de Engenharia Química da UFTM. A proposta surgiu como alternativa para um conjunto didático de baixo custo, que utiliza conceitos de balanços de massa e energia envolvidos no projeto de uma torre de resfriamento. A torre foi construída com altura de recheio de $1,29 \mathrm{~m}$, diâmetro de $0,25 \mathrm{~m}$, preenchida com recheio tipo grade trapezoidal e com custo final de $R \$ 2.000,00$. Foi estudada a influência da vazão e da temperatura de entrada da água no coeficiente volumétrico global de transferência de massa da fase gasosa e na eficiência da torre. Os resultados obtidos mostram que a eficiência da torre aumenta com a diminuição da vazão e diminui com o aumento da temperatura, enquanto o coeficiente volumétrico global diminui com o aumento da temperatura. Tais resultados estão em concordância com o desempenho de torres comerciais, possibilitando visualizar, na prática, a teoria sobre torres de resfriamento.

PALAVRAS-CHAVE: Psicrometria; Reuso de água; Transferência de calor e massa.

\section{DESIGN AND CONSTRUCTION OF A WATER-COOLING TOWER}

\begin{abstract}
The treatment of water in cooling systems affects the efficiency of production and the useful life of the equipment involved. In such processes, the high-water demand, the water is reused. To design equipment involving thermal exchanges, mass transfer coefficients must be estimated with reasonable precision, mass and energy balance equations are used and the heat and mass transfer coefficients must be estimated with reasonable precision, allowing the temperature gradients required for the best performance of the equipment to be reached. The aim of this study was to build and analyse the performance of a water-cooling tower for use in practical classes of the Chemical Engineering course at UFTM. The proposal emerged as an alternative to a low-cost teaching set, which use concepts of mass and energy balances involved in


the design of a cooling tower. The tower was built with a filling height of $1,29 \mathrm{~m}$, diameter of $0,25 \mathrm{~m}$, filled with trapezoidal grid-type filling and with a final cost of $R \$$ 2000,00 . The influence of water flow and inlet temperature on the global volumetric mass transfer coefficient of the gas phase and on the tower efficiency was studied. The results obtained show that the tower efficiency increases with decreasing flow and decreases with increasing temperature, while the global volumetric coefficient decreases with increasing temperature. Such results agree with the performance of commercial towers, making it possible to visualize, in practice, the theory of cooling towers.

KEYWORDS: Heat and mass transfer; Psychrometric; Water reuse.

\section{INTRODUÇÃO}

Estima-se que $97,5 \%$ do total de água está nos oceanos, enquanto $2,5 \%$ é água doce, sendo que menos de $0,01 \%$ é água potável (COSTA et al., 2020). Apesar de ser considerado um recurso renovável, a quantidade de água doce disponível em terra é limitada e sua qualidade está sob constante pressão, resultando em custos cada vez maiores de obtenção e processamento (CADORE et al., 2018).

A atividade humana e industrial vem gradativamente ampliando a capacidade de alteração do meio ambiente, principalmente no recurso água (WAN et al., 2020). Cada vez mais empresas buscam a modernização e 0 aumento em sua produtividade, e como resultado, mais água é necessária para a fabricação, geração de calor e refrigeração industrial. A Tabela 1 apresenta uma previsão do consumo de água para determinados processos de produção industrial.

TABELA 1. Volume de água consumido por unidade produzida.

\begin{tabular}{lc}
\hline Processo de Produção Industrial & $\begin{array}{c}\text { Consumo por Unidade } \\
\text { Produzida }\end{array}$ \\
\hline Fabricação de polpa de celulose & 15 a $200 \mathrm{~m}^{3} / \mathrm{t}$ \\
Branqueamento da polpa de celulose & 80 a $200 \mathrm{~m}^{3} / \mathrm{t}$ \\
Fabricação de papel & 30 a $250 \mathrm{~m}^{3} / \mathrm{t}$ \\
Fabricação de polpa e papel integrados & 200 a $250 \mathrm{~m}^{3} / \mathrm{t}$ \\
Fabricação de aço & 4 a $200 \mathrm{~m}^{3} / \mathrm{t}$ \\
Fabricação de cerveja & 8 a $13 \mathrm{l} / / \mathrm{produzido}$ \\
Fabricação de leite em pó & 0 a $18 \mathrm{~m}^{3} / \mathrm{t}$ \\
Processamento úmido de algodão & 80 a $600 \mathrm{~m}^{3} / \mathrm{t}$ \\
Abate de gado bovino & 0,2 a $9 \mathrm{~m}^{3} / \mathrm{cabeça}^{2}$ \\
\hline
\end{tabular}

Fonte: Von Sperling, M. (2005)

Para um consumo consciente de recursos hídricos, buscando aumento de eficiência e produtividade industrial, é necessário estabelecer novas ferramentas de gestão de recursos naturais. Sendo assim, processos que priorizam a reutilização da água vêm ganhando maior espaço dentro do mercado atual (POHL; LENZ, 2017).

O processo de reutilização de água é caracterizado pelo uso da água previamente utilizada, uma ou mais vezes, em certas atividades humanas, para atender às necessidades de outros usos benéficos (WU et al., 2019). Logo, o reuso da água é essencial, não apenas para sustentabilidade do planeta com seu uso consciente, mas também para a otimização dos processos, em que indústrias passam a reduzir sua captação de água nos corpos d'água naturais e a quantidade de efluentes a serem liberados posteriormente. 
Como alternativa para reutilização de água nos processos industriais, as torres de resfriamento tornam-se satisfatórias, pois são equipamentos de operação contínua, com aplicações no resfriamento da água que circula nas refinarias de petróleo, indústrias químicas, farmacêuticas, metalúrgicas e têxteis, estações de energia e refrigeração de edifícios (CREMASCO, 2015).

Essencialmente, uma torre de resfriamento é uma coluna de transferência simultânea calor e massa, que fornece uma grande área de contato entre a água a ser resfriada e o ar atmosférico. A água transfere calor residual para o ar atmosférico, sendo resfriada através do contato direto com o ar no recheio de enchimento interno da torre. O resfriamento da água ocorre por meio da transferência de calor latente (evaporação da água) e sensível (diferença de temperatura entre a água e o ar), que respondem respectivamente por $80 \%$ e $20 \%$ do calor total transferido (ALPINA, 2014).

A entrada de ar é realizada por circulação mecânica para o interior da torre, utilizando um ventilador na base da torre ou um exaustor na parte superior do sistema, aumentando assim o fluxo de ar que troca calor com a água. O sistema utiliza diversos componentes, como recheio, eliminador de gotas, ventilador, exaustor, bicos aspersores e outros (McCABE et al., 2005).

Devido à importância das torres de resfriamento para processos industriais, o seu estudo é abordado em cursos de engenharia, porém com certo grau de dificuldade didática por serem equipamentos de difícil acesso para aulas práticas (ONGARATTO et al., 2010). Neste contexto, o uso de protótipos de torres de resfriamento auxilia no processo de ensino-aprendizado, embora ainda sejam pouco utilizados por terem poucos modelos comerciais e de baixo custo (NARCIZO et al., 2018).

Portanto, o presente trabalho teve como objetivo construir um protótipo de uma torre de resfriamento de água, utilizando equações de balanços de massa e energia para cálculo dos coeficientes de transferência de calor e de massa. A construção do protótipo, suportada por conceitos teóricos bem fundamentados, pode propiciar significativos resultados no processo de ensino e aprendizagem.

\section{MATERIAL E MÉTODOS}

Inicialmente, realizou-se um estudo preliminar do funcionamento de uma torre de resfriamento de água, onde ocorre contato direto entre uma fase líquida, a água quente, e uma fase gasosa, 0 ar. Para esse contato ocorrer de maneira eficiente, o equipamento deve ser construído conforme esquema da Figura 1.

FIGURA 1. Esquema da torre de resfriamento.

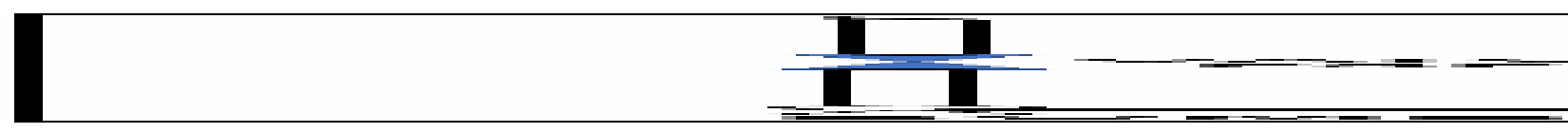

Fonte: Autores (2021) 
A água quente entra na torre pela parte superior e o ar atmosférico na parte inferior, ocorrendo escoamento em contracorrente entre as duas fases. $O$ recheio da torre permite que haja um contato mais eficiente entre as fases.

Para estabelecer a vazão da água, uma bomba é instalada, e o controle da vazão é realizado por uma válvula. $O$ ar é injetado na torre por um ventilador, ou motor de refrigeração. A água resfriada na torre cai no reservatório da água fria onde a bomba é instalada, sendo enviada para um aquecedor elétrico no topo da torre.

Para o dimensionamento da torre, balanços de massa e de energia são realizados no volume ocupado pelo recheio, com escoamento em contracorrente (Figura 2).

FIGURA 2. Nomenclatura usada no balanço de massa e energia na torre.

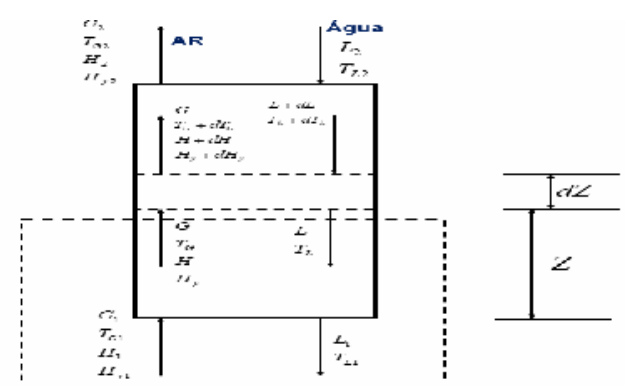

Fonte: Autores (2021)

A água quente é alimentada no topo da torre, na temperatura $T_{L 2}$ e vazão $L_{2}$, sendo distribuída de maneira uniforme através do recheio, que ocupa uma altura $Z$. $\mathrm{O}$ ar atmosférico, com fluxo ascendente, entra na base da torre na temperatura $T_{\mathrm{G} 1} \mathrm{e}$ vazão $G_{1}$. Logo que há o contato das fases água e ar no recheio, ocorrem transferências simultâneas de calor e de massa.

Realizando um balanço de energia numa espessura pequena $(\mathrm{dZ})$ do recheio, o calor transferido da água para o ar pode ser calculado usando a equação (1):

$$
q_{\text {AGUA }}=L c_{L}\left(T_{L}-T_{L 1}\right)
$$

$\mathrm{Na}$ equação (1), $\mathrm{L}$ é a vazão da água $(\mathrm{kg} / \mathrm{s}), C_{L}$ é o calor específico da água $(\mathrm{J} / \mathrm{kg} . \mathrm{K}), T_{L}$ é a temperatura da água na entrada do recheio $\left({ }^{\circ} \mathrm{C}\right)$ e $T_{L 1}$ é a temperatura da água na saída do recheio $\left({ }^{\circ} \mathrm{C}\right)$.

Para o ar que entra na parte inferior do recheio, o calor recebido é calculado pela equação (2) usando as entalpias na entrada e na saída:

$$
q_{A r}=G\left(H_{y}-H_{y 1}\right)
$$

$\mathrm{Na}$ equação (2), $\mathrm{G}$ é a vazão do $\mathrm{ar}(\mathrm{kg} / \mathrm{s}), \mathrm{H}_{\mathrm{y}}$ é a entalpia do ar na saída do recheio $(\mathrm{J} / \mathrm{kg})$ e $\mathrm{H}_{\mathrm{y} 1}$ é a entalpia do ar na entrada do recheio $(\mathrm{J} / \mathrm{kg})$. A equação (3) 
apresenta o balanço de energia na torre, considerando que o calor transferido pela água é absorvido pelo ar no espaço ocupado pelo recheio:

$$
L c_{L}\left(T_{L}-T_{L 1}\right)=G\left(H_{y}-H_{y 1}\right)
$$

O ponto relevante para uso da equação (3) consiste na determinação da curva de equilíbrio entre as duas fases, que descreve a relação entre a entalpia da mistura ar-água com a temperatura. Os valores dessa relação estão na Tabela 2.

TABELA 2. Entalpias da mistura ar x vapor d'água.

\begin{tabular}{ccccccccccc}
$\mathrm{T}\left({ }^{\circ} \mathrm{C}\right)$ & 15,6 & 26,7 & 29,4 & 32,2 & 35,0 & 37,8 & 40,6 & 43,3 & 46,1 & 60,0 \\
$\mathrm{H}_{\mathrm{y}}$ & 43,6 & 84,0 & 97,2 & 112,1 & 128,9 & 148,2 & 172,1 & 197,2 & 224,5 & 461,5 \\
$(\mathrm{~kJ} / \mathrm{kg})$ & & & & & & & & & & \\
\hline
\end{tabular}

Fonte: Perry; Green (2018)

Medindo a temperatura do ar na entrada da torre, sua entalpia $H_{y 1}$ é determinada na carta psicrométrica. Estabelecendo um valor desejado para a temperatura da água resfriada, $T_{L 1}$, a equação (4) fornece o valor da entalpia do ar $\mathrm{H}_{\mathrm{y} 2}$ na saída do recheio:

$$
\frac{L c_{L}}{G}=\frac{\left(H_{y_{2}}-H_{y 1}\right)}{\left(T_{L 2}-T_{L 1}\right)}=\alpha
$$

A equação (4) mostra que uma reta de inclinação $\alpha$ pode ser traçada no diagrama da entalpia $x$ temperatura, partindo das correntes de água e ar na parte inferior do recheio, no ponto de coordenadas $\left(T_{L 1}, H_{y 1}\right)$, até a saída do recheio, no ponto com coordenadas ( $\left.\mathrm{T}_{\mathrm{L} 2}, \mathrm{H}_{\mathrm{y} 2}\right)$.

A equação de transferência de calor na fase gasosa pode ser escrita usando - coeficiente volumétrico global de transferência de massa da fase gasosa, conforme apresentado pela equação (5) para o ar.

$$
G\left(H_{y}-H_{y 1}\right)=M_{B} K_{G} a P d Z\left(H_{y 2}-H_{y 1}\right)
$$

$\mathrm{Na}$ equação (5), a grandeza $\mathrm{K}_{\mathrm{G}} \mathrm{a}$ é o coeficiente volumétrico global de transferência de massa da fase gasosa $\left(\mathrm{kmol} / \mathrm{s} \cdot \mathrm{m}^{3} \cdot \mathrm{Pa}\right)$.

O resultado apresentado pela equação (5) pode ser aplicado desde o ponto de coordenadas $\left(T_{L 1}, H_{y 1}\right)$, na reta operacional do sistema, até o ponto de coordenadas $\left(\mathrm{T}_{\mathrm{L} 2}, \mathrm{H}_{\mathrm{y} 2}\right)$, encontrando todos os pontos que descrevem a transferência simultânea de calor e de massa na altura de recheio da torre.

Aplicando a equação (5) do ponto de coordenadas $\left(T_{L 1}, H_{y 1}\right)$ na parte inferior do recheio, até o ponto na saída do recheio, a altura $Z$ ocupada pelo recheio pode ser calculada, conforme a equação (6):

$$
Z=\frac{G}{k_{G} a M_{B} P} \int_{H_{y 1}}^{H_{y 2}} \frac{d H_{y}}{\left(H_{y i}-H_{y}\right)}
$$




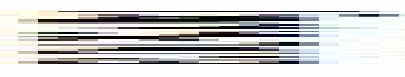

\section{Construção e Otimização do Equipamento}

Com a estimativa inicial da altura e do diâmetro interno, fez-se um desenho do equipamento para visualização dos componentes necessários (Figura 3).

FIGURA 3. Desenho Esquemático da Torre de Resfriamento.

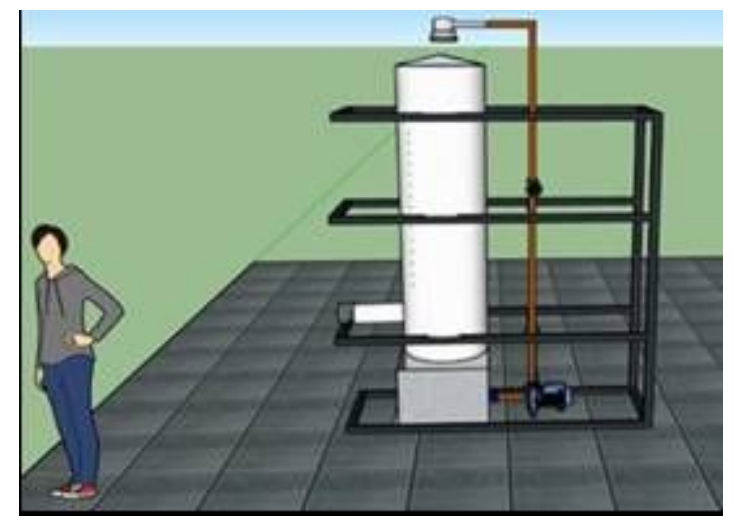

Fonte: Autores (2021)

A construção da torre iniciou-se pelo suporte de sustentação do equipamento, feito de metallon. Para o reservatório de água, foi escolhido um reservatório de plástico de 150 litros. Foi acoplada ao suporte/base de matallon, uma tubulação de PVC com $30 \mathrm{~cm}$ de diâmetro interno e com altura de projeto de 2 metros. Fez-se uma base para acoplar uma tubulação de PVC com diâmetro interno de $15 \mathrm{~cm}$ e comprimento de $20 \mathrm{~cm}$, na qual posicionou-se o motor de refrigeração para fornecimento de ar.

Logo após a finalização de toda estrutura para suspensão do circuito de tubulação de PVC, por onde a água circula, foram anexadas as tubulações de PVC de diâmetro 3/4", assim como o preenchimento da torre com recheio selecionado (Enchimento tipo Grade Trapezoidal TEG17 em polipropileno PP- Termoparts), um dos mais vendidos para torre de resfriamento no país por apresentar alta qualidade e ótimo desempenho (LI et al., 2017).

Foi confeccionado também um distribuidor de líquido tipo ralo, posicionado no topo da torre de resfriamento, para garantir que a água que escoa a partir do chuveiro seja dispersada por toda superfície interna do recheio da torre. A Figura 4 
mostra o enchimento escolhido e o distribuidor de líquido na parte superior do equipamento.

FIGURA 4. a) Enchimento tipo Grade Trapezoidal TEG17 em polipropileno (PP Termoparts) e b) distribuidor de líquido.

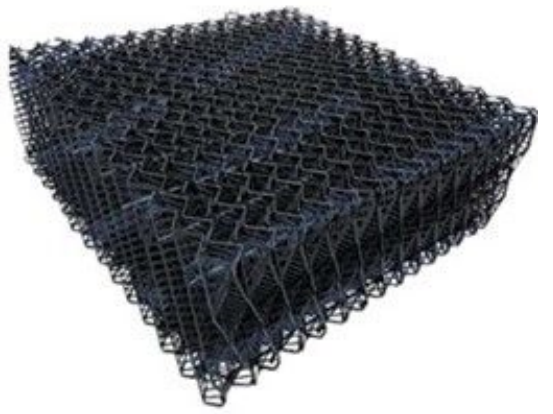

(a)

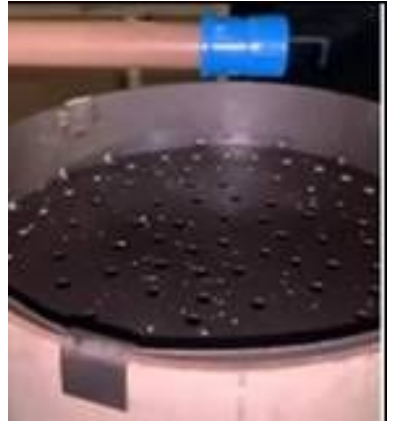

(b)

Fonte: Autores (2021)

Imediatamente depois foi feita a confecção da base para sustentar a bomba de circulação de água, seguida de sua instalação na rede de tubulação de PVC. Foi instalada uma válvula para controlar a vazão da água. Fez-se a ligação elétrica da bomba e do disjuntor do chuveiro. A Figura 5 mostra as partes instaladas.

FIGURA 5. Partes constituintes da torre: (a) - parte superior; (b) - bomba instalada.

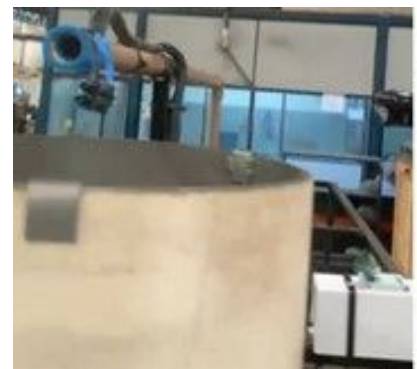

(a)

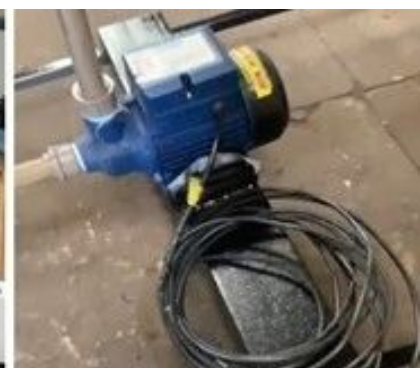

(b)

Fonte: Autores (2021)

Por fim, foi realizada a pintura de toda estrutura para evitar desgaste ao longo do tempo. Foram instalados o chuveiro e o motor de refrigeração. A torre de resfriamento construída no trabalho é apresentada na Figura 6. 
FIGURA 6. Torre de resfriamento construída: (a) - Vista frontal; (b) - Vista lateral.

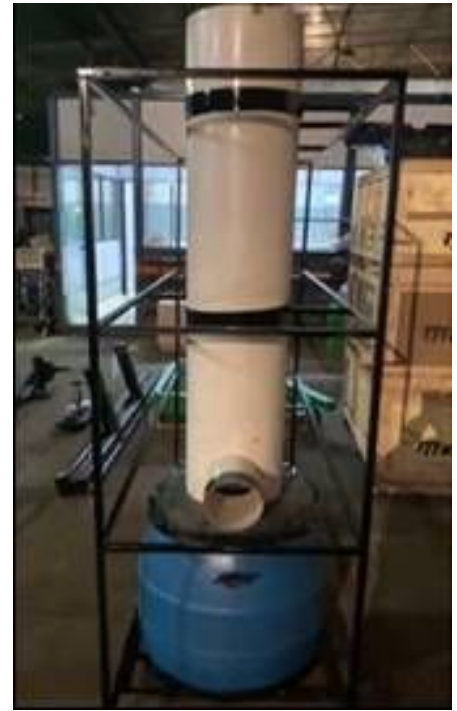

(a)

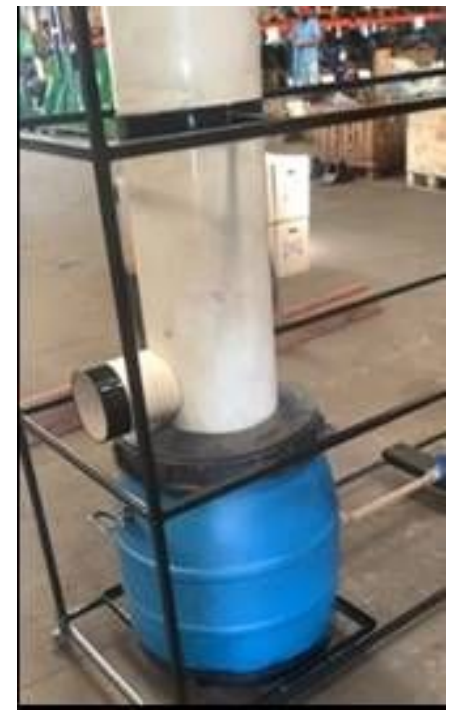

(b)

Fonte: Autores (2021)

A torre de resfriamento foi construída usando os seguintes componentes:

- 01 Tubo de PVC Diâmetro Externo = 250mm, 2 metros de comprimento;

- 01 Tubo de PVC Diâmetro Externo = 150mm, 200 mm de comprimento;

- 01 Reservatório de água 150 Litros;

- Tubulação PVC $3 / 4$ " de diâmetro;

- 06 Adaptadores curtos 3/4";

- 02 Bucha de Redução 1" para 3/4" soldável;

- 02 Cotovelos 90 3/4";

- 01 Adesivo PVC Bisnaga 17gramas;

- 01 Válvula esfera de Alavanca em aço 3/4";

- 01 Registro de esfera $3 / 4$ ";

- 01 Malha de compressão da água junto com chapa trava malha;

- Kit (100 peças) arrebite $4 \mathrm{~mm}$;

- 01 Bomba modelo ICS-500AB 1/2cv Bivolt;

- 01 Chuveiro Elétrico de Plástico 220V;

- $1 \mathrm{~m}^{3}$ Recheio de enchimento de contato tipo filme TEF-19 ( $\mathrm{D}=300 \times \mathrm{x} A 00 \mathrm{~mm}$ );

- 01 Distribuidor de líquido (peneira);

- 05 Barras de Metallon 20mmx20mmx2mm;

- 04 Rodas para transporte do equipamento (2 rodas com trava e 2 rodas padrão);

- 01 Disjuntor 32 Ampares;

- 02 Conector Borne $6 \mathrm{~mm}$;

- 02 Plug macho $2 \mathrm{P}+\mathrm{T} 20^{\circ}$ Reto;

- 05 Cabo PP 2 x 4 m;

- 01 Centro EXT 02 Disj Din Steck;

- 01 Motor de Refrigeração. 
Um parâmetro importante na análise do funcionamento de uma torre de resfriamento de água, é a eficiência da torre, , que representa o quociente entre a taxa de calor removida pela torre e a máxima taxa de calor possível, relacionada à temperatura de bulbo úmido do ar que entra na torre. Valores considerados razoáveis estão no intervalo entre 0,30 até 0,85 (LLANO-RESTREPO; MONSALVEREYES, 2017). Esse intervalo depende do recheio da torre e da velocidade de entrada do ar na base da torre.

\section{RESULTADOS E DISCUSSÃO}

Para analisar o funcionamento da torre construída neste trabalho, foram realizados experimentos com os seguintes parâmetros de entrada: vazão de água $(L)$, vazão de $\operatorname{ar}(G)$ e temperatura de entrada da água $\left(T_{L 2}\right)$ no topo da torre. Os valores selecionados para esses parâmetros foram estabelecidos com base nas limitações da bancada construída.

Durante a realização dos experimentos foram coletados os seguintes dados: temperatura de bulbo seco e de bulbo úmido do ar na entrada da torre, e temperatura de saída da água da torre $\left(T_{L 1}\right)$. Com esses valores e aplicando a metodologia descrita anteriormente, os dois principais parâmetros relacionados ao desempenho da torre, o coeficiente volumétrico global de transferência de massa da fase gasosa $\left(\mathrm{K}_{\mathrm{G}} \mathrm{a}\right)$ e a eficiência ( ) da torre foram calculados (LLANO-RESTREPO; MONSALVE-REYES, 2017). Os resultados encontrados permitiram avaliar a influência da vazão e da temperatura de entrada da água no comportamento de tais parâmetros.

\section{Influência da vazão na eficiência da torre}

A Figura 7 mostra a variação da eficiência da torre ( ) com a razão entre as vazões da água e do ar na entrada da torre.

FIGURA 7. Influência das vazões na eficiência da torre

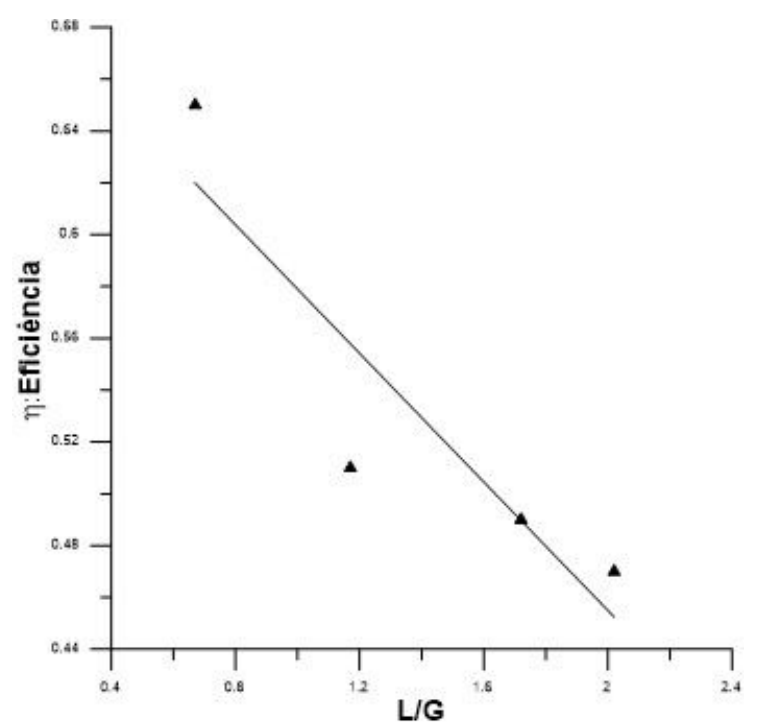

Fonte: Autores (2021).

Nota-se que o aumento da vazão da água que entra pelo topo da torre provoca uma diminuição na eficiência da torre, pois aumentando a vazão de água, a área de contato necessária para a convecção e para a evaporação é reduzida, 
resultando no aumento da temperatura de saída da água e redução das taxas de transferência de calor. Esses resultados estão de acordo com os que foram encontrados por Enayatollahi et al. (2017), que analisaram torres de resfriamento operando com recheios semelhantes ao utilizado na construção da torre.

\section{Influência da temperatura de entrada da água na eficiência da torre}

A Figura 8 mostra a variação da eficiência da torre com a temperatura de entrada da água.

FIGURA 8. Influência da temperatura de entrada da água na eficiência da torre.

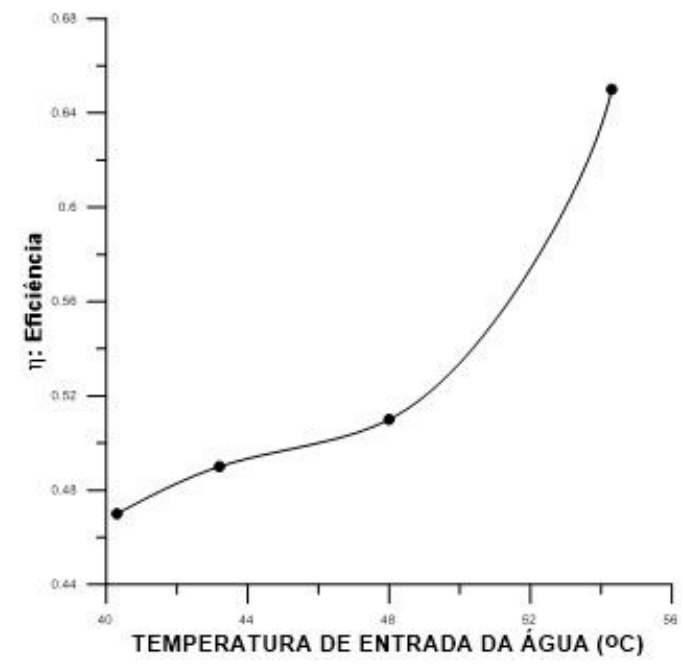

Fonte: Autores (2021).

Pode ser observado na Figura 8 que a eficiência da torre aumenta com o aumento da temperatura da água de entrada. Isto ocorre porque o aumento da temperatura aumenta também o gradiente de transferência de calor da torre. Como o resfriamento é uma operação controlada simultaneamente pelas transferências de calor e de massa, um aumento do gradiente de temperatura ocasiona aumento na transferência de calor e consequente aumento da transferência de massa, aumentando assim a eficiência global do sistema. Estes resultados estão de acordo com os encontrados por Llano-Restrepo e Monsalve-Reys (2017), que mostra que a eficiência de uma torre de resfriamento aumenta com a temperatura e diminui com a vazão.

\section{Influência da temperatura de entrada da água no coeficiente volumétrico de transferência de massa}

Os resultados experimentais obtidos permitiram o cálculo do coeficiente $\mathrm{K}_{\mathrm{G}} \mathrm{a}$ através da Equação (6). A Figura 9 mostra como a temperatura de entrada da água afeta o valor do coeficiente. 
FIGURA 9. Influência da temperatura de entrada da água no coeficiente volumétrico de transferência de massa.

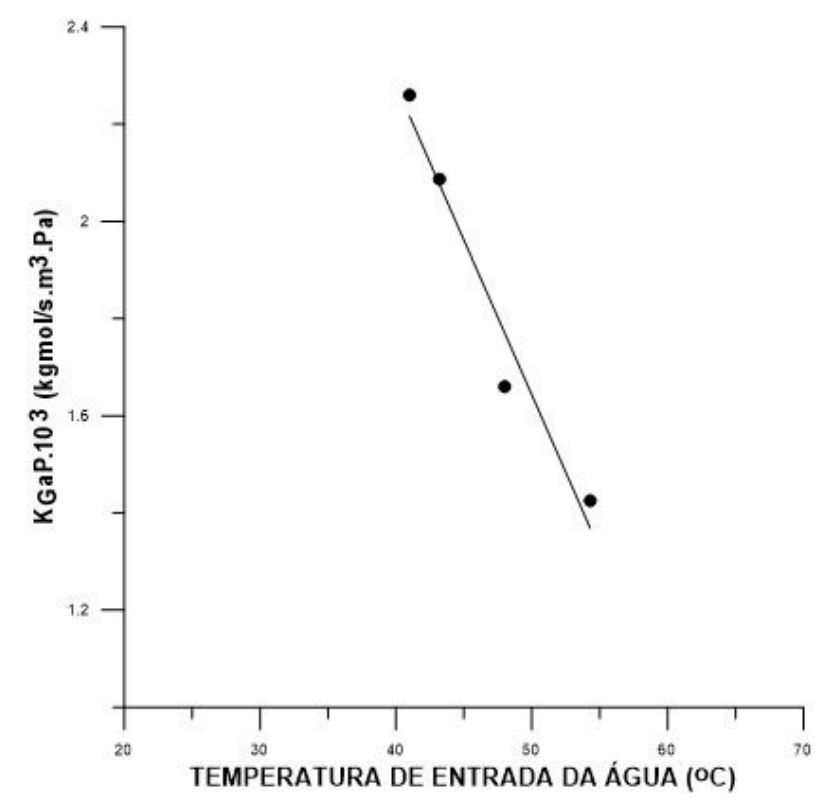

Fonte: Autores (2021).

Nota-se que o coeficiente volumétrico global de transferência de massa aumenta com a diminuição da temperatura da água. Uma explicação para esse comportamento está relacionada com o aumento da espessura da camada limite onde ocorre a transferência (GEANKOPLIS et al., 2018). Uma camada limite mais espessa dificulta a transferência de massa na fase gasosa, e isto diminui o coeficiente volumétrico.

\section{CONCLUSÃO}

A construção de uma torre de resfriamento de água foi realizada com um custo final de $R \$ 2000,00$, resultando no conjunto didático que possibilitou visualizar, na prática, o que se aprende na teoria sobre resfriamento de água utilizando as equações de balanços de massa e energia.

Os resultados obtidos foram utilizados para determinar a eficiência da torre e calcular o coeficiente volumétrico global de transferência de massa. $O$ estudo mostrou que a eficiência da torre aumenta com o aumento da temperatura da água e diminui com o aumento da vazão, enquanto o coeficiente volumétrico global de transferência de massa, obtido para o tipo de recheio utilizado na torre, diminui com o aumento da temperatura da água. Esses resultados estão em concordância com o desempenho de torres de resfriamento com recheio, conforme indicam trabalhos científicos já publicados.

Com isso, o conjunto didático elaborado permite realizar uma aula prática de uma operação unitária, onde é possível visualizar a influência de parâmetros operacionais no desempenho do equipamento e demonstrar a aplicabilidade das equações de balanços de massa e de energia aprendidas nas aulas teóricas. 


\section{REFERÊNCIAS}

ALPINA. Catálogo Técnico - Torres de Resfriamento ALPINA - Modelos 180/2 a 550/5. São Paulo, 2014. Disponível em: www.alpina.com.br. Acesso em: 29 mai. 2021.

CADORE, I. R.; SILVA, M. K.; POLLO, L. D.; TESSARO, I. C. Wastewater treatment in a pilot-scale submerged membrane bioreactor: study of hydrodynamics under constant operating pressure. Brazilian Journal of Chemical Engineering, v.35, n.01, p. 51-61, january-march, 2018. dx.doi.org/10.1590/01046632.20180351s20160416

COSTA, D. M.; SILVA, J. L. S.; BARRETO, M. S. O. D.; BARRETO, G. C. Reúso de Efluentes tratados para fins de operação de torres de resfriamento. Brazilian Applied Science Review, Curitiba, v. 4, n. 4, p. 2568-2577, Jul./Ago. 2020. DOI: 10.34115/basrv4n4.032, ISSN 2595-3621

CREMASCO, M. A. Fundamentos de transferência de massa, $3^{a}$ ed. São Paulo: Edgard Blücher, 2015. 460 p. ISBN: 978-85-212-0904-1

ENAYATOLLAHI, R.; NATES, R. J.; ANDERSON, T. Characterising the heat and mass transfer coefficients for a crossflow interaction of air and water. International Journal of Heat and Mass Transfer, v. 111, p. 94-104, august 2017. https://doi.org/10.1016/jijheatmasstransfer.2017.03.098

GEANKOPLIS, C. J.; HERSEL, A. A.; LEPEK, D. H. Transport Processes and Separation Process Principles, $5^{\text {th }}$ ed. Pearson PTG, 2018. ISBN: $9780134181028,0134181026$.

LLANO-RESTREPO, M.; MONSALVE-REYES, R. Modeling and simulation of counterflow wet-cooling towers and the accurate calculation and correlation of mass transfer coefficients for thermal performance prediction. International Journal of Refrigeration 74, p. 47-72, 2017.

http://dx.doi.org/10.1016/j.jirefrig.2016.10.018

LI, X.; GURGENCI, H.; GUAN, Z.; WANG, X.; DUNIAM, S. Measurements of crosswind influence on a natural draft dry cooling tower for a solar thermal power $\begin{array}{lllll}\text { plant. Applied } & \text { Energy 2017. }\end{array}$ http://dx.doi.org/10.1016/j.apenergy. 2017.10.038

McCABE, W. L..; SMITH, J. C.; HARRIOT, P. Unit Operations of Chemical Engineering. $7^{\text {th }}$ ed. New York. McGraw-Hill Education, 2005. ISBN-13: 9780072848236.

NARCIZO, R. B.; SOUZA, F. E.; OLIVEIRA, C. M.; HOTTZ, C. M.; VARGAS, M. S. N.; SILVA, M. S. A prototipação de baixo custo como ferramenta pedagógica no ensino de engenharia do produto. Revista de Ensino de Engenharia, v. 37, n. 3, p. 64-72, 2018. ISSN 2236-0158 - DOI: 10.5935/2236-0158.20180031 
ONGARATTO, R. S.; SARKISB, J. R.; RECH, R. Construção de uma torre de resfriamento de bancada para o ensino de operações unitárias. Revista de Ensino de Engenharia, v. 29, n.2, p. 24-34, 2010 - ISSN 0101-5001. DOI: 10.15552/22360158/abenge.v29n2p27-34

PERRY, R. H.; GREEN, D. W. Perry's Chemical Engineers' Handbook. $9^{\text {th }}$ ed., New York: McGraw-Hill, 2018. ISBN-13: 978-0071834087.

POHL, S. C.; LENZ, D. M. Utilização de efluente tratado em complexo industrial automotivo. Engenharia Sanitária e Ambiental, v. 22, n. 3, p. 551-562, maio/jun 2017. DOI: 10.1590/s1413-41522016122371

PÓOS, T.; VARJU, E. Mass transfer coefficient for water evaporation by theoretical and empirical correlations, v. 153, International Journal of Heat and Mass Transfer, 2020, 119500. https://doi.org/10.1016/j.ijheatmasstransfer.2020.119500

RODRIGUES, L. G. G.; PARISSOTO, E. I. B.; CARCIOFI, B. A. M.; LAURINDO, J. B. Experimental approach to assess evaporative cooling under forced air flow. Brazilian Journal of Chemical Engineering, v. 34, n. 01, p. 171-181, january-march, 2017. dx.doi.org/10.1590/0104-6632.20170341s20150433

VON SPERLING, M. Introdução à Qualidade das Águas e Ao Tratamento de Esgotos, v. 1, $3^{\mathrm{a}}$ ed., Ed. DESA, 2005.

WAN, Y.; SOH, A.; SHAO, Y.; CUI, S.; TANG, Y.; CHUA, K. J. Numerical study and correlations for heat and mass transfer coefficients in indirect evaporative coolers with condensation based on orthogonal test and CFD approach. International Journal of Heat and Mass Transfer, v. 153, June 2020, 1195580. https://doi.org/10.1016/j.ijheatmasstransfer.2020.119580

WU, T.; WEI, H.; GE, Z.; YANG, L.; DU, X. Cooling water mass flow optimization for indirect dry cooling system of thermal power unit under variable output load, International Journal of Heat and Mass Transfer, v. 133, p. 1-10, April 2019. https://doi.org/10.1016/j.ijheatmasstransfer.2018.11.097 